\section{JIBM}

Journal of International Business and Management (JIBM) Journal Homepage: https://rpajournals.com/jibm

\title{
Justification for Adopting Qualitative Research Method, Research Approaches, Sampling Strategy, Sample Size, Interview Method, Saturation, and Data Analysis
}

\author{
Md Asadul Islam" \\ Faraj Mazyed Faraj Aldaihani \\ Swinburne University of Technology, (Sarawak Campus), Malaysia \\ Universiti Putra Malaysia (UPM), Malaysia
}

\begin{abstract}
Justifying the adoption of the qualitative research method to satisfy the examiners (for thesis) and reviewers (for journal articles) is a challenging task for researchers in business, management, marketing, tourism, hospitality and albeit in social science domain. The difficulty continues in establishing the justification for selecting qualitative research approaches, sample strategy, sample size, data collection methods (i.e. interview methods), saturation, and data analysis. In this guide, we aim to ground brief justifications for researchers and guidance on how to justify the section of qualitative research method in thesis and journal articles. This study also provides brief justification on selecting specific qualitative research approaches, sampling strategies, sample size, interviews, and data analysis methods. Furthermore, this study provides a glimpse of justification regarding when and how to reach saturation point in qualitative research.

Keywords: Qualitative research method, Research approaches, Sampling strategy, Sample size, Interview method, Saturation, and Qualitative data analysis (QDA)

*Corresponding author: Md Asadul Islam; Email: mislam@swinburne.edu.my DOI: https://doi.org/10.37227/JIBM-2021-09-1494
\end{abstract}

\section{Introduction}

A number of researchers, especially novice researchers, encounter difficulties in understanding qualitative research, its approaches, sampling strategies, data collection methods, data analysis strategies, how and when to reach interview saturation points and so on. However, many try to overcome these challenges and finally develop a qualitative study. There are numerous books and articles relating to the qualitative method as it is very significant in educational research because of its capability to address research questions regarding 'how' and 'why' (Cleland, 2017). However, novice researchers sometimes lose their interest in qualitative research owing to a lack of understanding of the critical approaches. Furthermore, lengthy explanations of the methods in different books and articles also sometimes demotivate researchers from adopting qualitative research (Anderson, 2010). Therefore, researchers of this study aimed to briefly present the justification for adopting the key areas of the qualitative methods. As it is fairly challenging to incorporate all the tools associated with qualitative research method, the key areas that have been discussed in this 
study include research approaches, sampling strategies, sample size, interview methods, saturation, and data analysis methods.

\section{What is Qualitative Research? And Why?}

We first present a definition of qualitative research. We then provide you with some examples to apply this definition of qualitative research to understand what this method is. Research method that allows one to explore or investigate the quality of relationships, activities, situations, or materials is mainly known as qualitative research (Fraenkel, Wallen \& Hyun 2015). Consider the example of basic training on a machine in a factory i.e. readymade garment (RMG) factory. How do trainers train the employees? What are jobs employees do? What are the things that they perform as they have to meet target? In what type of teams do they have to engage? The list of questions is endless. Now, if you would like to achieve some insights about these concerns, you may try to portray or write the everyday experiences of the employees of the factory. Your focus would be only the employees of the particular factory or only particular employees eligible for the training. Hence, to explore or investigate the answer to those questions, you may observe the employees' activities in the factory on a regular basis if possible and try to describe what they do as elaborately as possible. Now, imagine what is your interest to the employees? The answer to this question is that preceding examples directly point to the fact that you are interested in the 'quality' of a particular activity than how often it occurs or how it would otherwise be evaluated. Therefore, to get your interest into reality, you will have to 'explore' the activities, situations, materials, and quality of relationships associated with those employees. This form of investigation or exploration is known as qualitative research.

Let us provide you further clarification adopting the views of Cleland (2017), who argued that qualitative research addresses the research questions concerning 'how' and 'why' and allows a researcher to understand the context, phenomena, and experiences. From this definition, you should understand that it is not easily possible to put the answers of 'how' and 'why' into numbers to understand the phenomena, context, objects, and experiences. However, getting answers to those 'how' and 'why' or any other 'Wh-questions' are essential and practical because those answers can extend the current knowledge, enrich existing literature, and further develop our understanding. So, what is the method, in general, that you should adopt/apply in your thesis or research paper in answering the questions of 'how' and 'why' or any other Wh-questions when you found it is not possible to easily put the answers in numbers? In this regard, the answer is that you should adopt qualitative research.

Let us further explain, when you should adopt qualitative research. You have to conduct qualitative research when you need to write something in a literary and flexible style that outlines stories or events, poems, or theatre without any restrictions of formal academic writing structure (Creswell \& Poth, 2018). Furthermore, you can adopt qualitative research to develop new theories, strengthen current theories, or even apply existing theories in new settings. Again, qualitative research is recommended when you identify inadequate or partial theories existing for a particular sample or geography or context. Moreover, you are suggested to adopt qualitative research when you recognise that current theories do not adequately capture specific issues or problems. Finally, you have to conduct qualitative research when you need to understand the settings or contexts in which respondents or participants or objects can address/outline an issue or problem. Qualitative research allows readers to know something new and probably find viable solutions to a problem. 


\section{Research Approach}

For explaining the qualitative research approach, we have selected the five research approaches proposed by Creswell and Poth (2018): narrative, phenomenology, grounded theory, ethnography, and case study. Identifying a research approach in qualitative research is critical for presenting a qualitative research paper, thesis, or dissertation as a sophisticated and structured study, which is mostly admired by the reviewers or supervisors. If you are still in doubt regarding the necessity of research approaches, we are describing two scenarios (adopted from Creswell \& Poth, 2018) for your understanding.

1. First scenario: There is a researcher who does not adopt a specific approach to qualitative research he or she is using. However, the study can be simple and might be limited to data collection using face-to-face interviews. He or she might have presented the data by thematic analysis; however, the analysis is not systematic or organised, and those themes are not presented chronologically.

2. Second scenario: A researcher has selected a specific approach to qualitative research, for example, phenomenological research approach. In this regard, in the method section of the study, he or she has provided a detailed explanation of the meaning of such an approach, how it informs, the procedure, and why it is being used. As a result, the results from this study are clear, chronological, and specific.

Which scenario would you find to be most organised, scholarly, inviting, and sophisticated? Your answer should be the second one. Hence, adopting a research approach in qualitative research is essential. However, in selecting one of the research approaches, your research focus and research problem can help most because the problem statement grounds the reason for selecting one or more than one approaches in qualitative research. Hence, see the following figure to determine your research approach for your research paper or thesis.
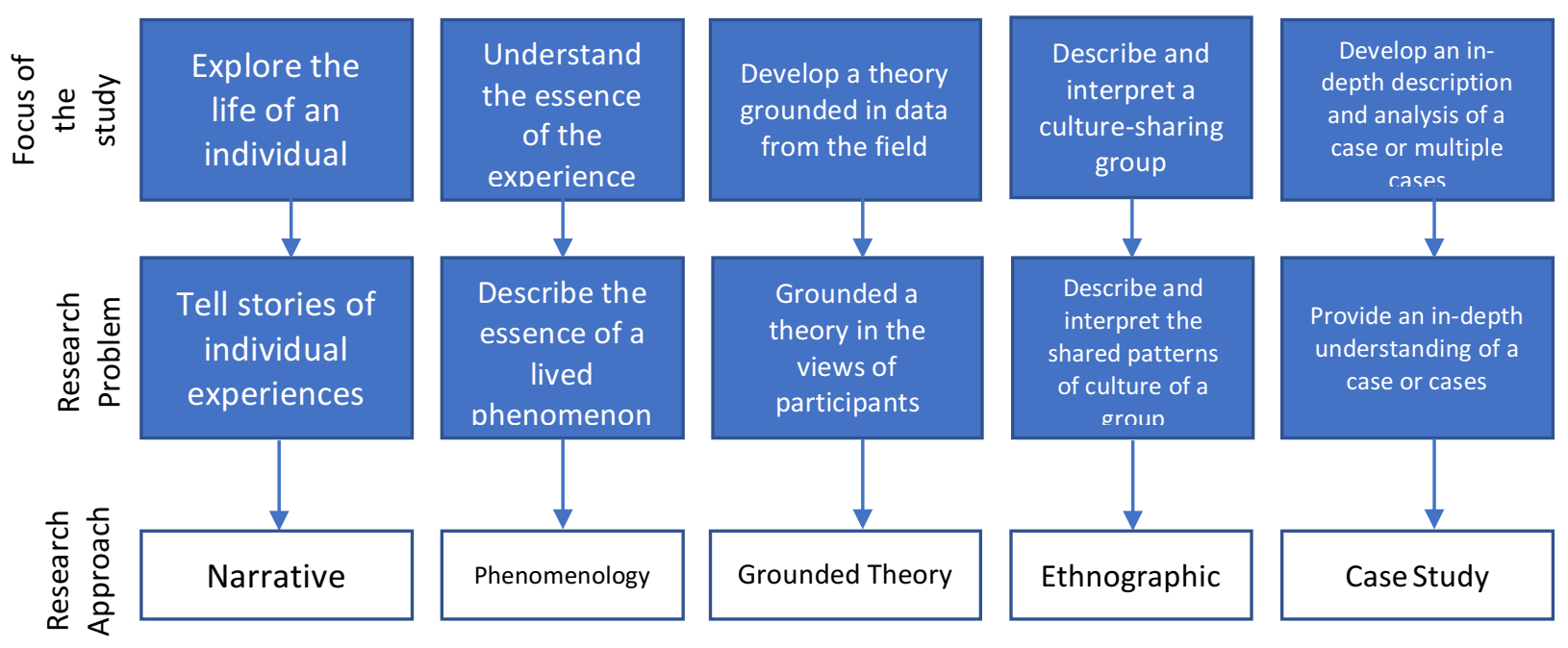

Figure 1: Qualitative Approaches

Source: Creswell and Poth (2018)

Figure 1 illustrates how you can select your research approach to qualitative research. However, it is important to first understand the primary focus of the study and research problem (i.e. problem statement) before you select or adopt one of the research approaches. 
Therefore, careful attention to the development of the strong focus of your study and research problem is highly recommended to select the most appropriate approach.

\section{Sampling Strategy}

There are many sampling strategies, and it is sometimes nearly impossible for novice researchers to identify and finalise one sampling strategy. So how can you select one and justify the adoption of the research? In this section, we tried to give some valuable insights for your understanding regarding the tactics and some sampling strategies commonly used in qualitative research so that you can select the most appropriate sampling strategies.

Sampling is the process of selecting the objects, participants, or respondents for your study. The participants or respondents or objects can be questioned, surveyed, or observed. However, a sample is a group on which information is obtained from a relatively larger population. A research population is also called an extensive/large collection of objects or individuals (i.e. respondents or participants) that is the central focus of a research investigation or query. Moreover, the research population - either objects or individuals are mainly identified to have similar characteristics. However, the larger group to which a researcher wishes to apply the results is known as the population (Fraenkel et al., 2015).

Like quantitative researchers, qualitative researchers usually cannot include a bigger sample from the population through the sampling strategies. It is because data amassed by qualitative researchers cannot be quantified; hence, such researchers try to reach a saturation point (discussed in the later section of this article). Therefore, it is vital to apply an appropriate sampling strategy to include the right participants, respondents, or objects for the qualitative research.

However, before we discuss some sampling strategies suitable for qualitative research, we should inform you that generalisation of the work to the whole research population is not the goal of most qualitative researchers (Dawson, 2019). They rather usually seek to explore, explain, or describe what is happening within a smaller group of people or objects (Dawson, 2019). Qualitative researchers do this because they believe data from the smaller group might provide insights into the behavior or situation of the broader research population. Those insights can be tested in future research, for example, in quantitative studies. If you adopt qualitative research, you have to accept that everyone is different; therefore, if you do the same research with another group of people, the results might not be the same.

However, there is a significant difference among the scholars regarding the sampling strategies for qualitative research. However, if you are trying to write a research paper, thesis, or dissertation, you should not be worried regarding the types of sampling strategies. You should be concerned about selecting the right one for your study. In this regard, you are recommended to read some scholarly documents where various types of sampling strategies are clarified (see e.g. Fraenkel et al., 2015; Saunders et al., 2009). However, the most common sampling strategies used in qualitative research are purposive sampling and convenience sampling. The former allows you to select objectives and specially qualified respondents or participants to collect the requisite data (Fraenkel et al., 2015).

The latter, on the other hand, allows selecting objectives and easily accessible respondents or participants to collect the requisite data. However, both of these sampling strategies are also called non-random sampling strategies. Moreover, purposive sampling was also divided into 16 types in the study of Patton (1990). Now, you may wonder that there are so many sampling strategies to know. Yes, you should know that would be great. However, we urge the researchers working on thesis or scholarly articles to read academic 
research journal articles related to the topic so that a suitable sampling can be identified and applied for data collection. This would allow them to justify why they adopted the sampling strategy.

\section{Data Collection Methods (Interview Method)}

There are a number of data collection methods available for a qualitative researcher, for example, interviews, observations, focus group discussion (FGD), textual, and visual analysis (Silverman, 2000; Gill et al., 2008). However, the most common data collection methods in qualitative research relating to business, management, marketing, or other social science studies are interviews and focus groups (Merriam \& Tisdell, 2015; Patton, 2015). However, we aim to present the major areas of interview methods so that a researcher knows how these methods work in practice and when their usages are appropriate in respective research.

The purpose of the interview is to explore the experiences, views, opinions, ideas, beliefs, or/and motivations of entities/individuals on particular objects, issues, or phenomena. In this regard, interviews mainly provide 'deeper' ideas or understanding of social issues or phenomena. Therefore, the interview method is the most appropriate when researchers are little known to one or more than one study phenomenon or issue. Furthermore, interview methods are also suitable for researchers when the topics are sensitive and participants or respondents want to verbally express their opinions or ideas.

However, there are mainly three types of interviews used in qualitative research: structured, semi-structured, and unstructured. Structured interviews allow a researcher to prepare administered questionnaires where predetermined questions (for respondents) are enumerated with no scope of follow-up questions. Though it is easy to manage, it does not offer the opportunity for deeper research to explore the issue or phenomenon.

On the other hand, an unstructured interview does not reflect any preconceived ideas or theories and is conducted with little or no organisation (May, 1991). Hence, researchers can easily start this interview with an opening question related to the research topic then the researcher can continue asking questions mainly based on the opening answer. However, this interview method can be lengthy and complicated to manage. Furthermore, since no predetermined questions are prepared in unstructured interviews, the researcher may lose the sequence of the questions under the topic. As a result, the participant may feel confused and eventually lost. The analysis of the collected data through this interview method could also be difficult for researchers, especially novice researchers, because this type of interview takes a long time. Therefore, if the researcher is not an expert, we do not recommend this interview method to be applied in the qualitative research. 


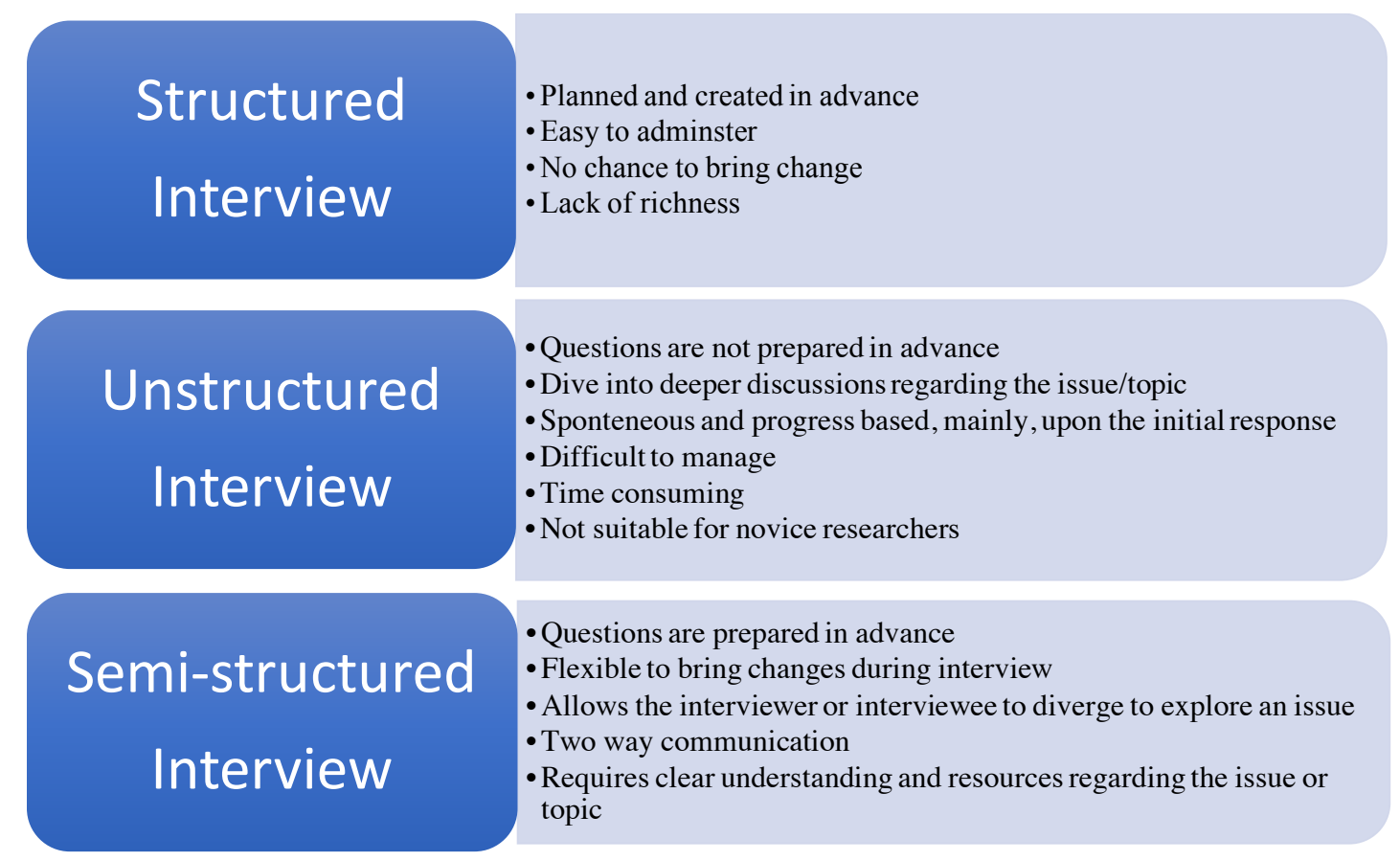

Figure 2: Various Types of Interviews

Source: Authors

The semi-structured interview allows a researcher to include some key questions relating to the topic; it further allows both interviewer or interviewee to diverge to explore an issue or response in deep (Schmidt, 2004; Saunders et al., 2009; Evans \& Lewis, 2018). Thus, this is flexible in comparison to structured interviews. The semi-structured interview allows the researcher to suggest or consult the participant (i.e. an interviewee) on what to talk about, especially when the interviewee digresses from the original topic. Thus, this is a helpful method for both interviewer and interviewee to confine themselves to the topic domain. This interview method creates a two-way communication between the interviewer or interviewee; therefore, open-ended responses can be taken, which allow the former to gather more indepth information relating to the research topic or issue. Our observation on the business, management, marketing, and even in social science research (academic research papers) allows us to assert that semi-structured interviews are the most widely used qualitative research data collection methods. More particularly, most novice researchers use this method to ensure that they can explore the issue by managing the interviews successfully. Therefore, we recommend the semi-structured interview as the most useful interview method for rich data exploration. However, it is vital to mention that the length of the interviews vary as per the requirements of the researcher, participants, and the research theme.

\section{Qualitative Data Analysis}

Qualitative data analysis (QDA) includes the interpretation, identification, and examination of patterns and themes in textual data and determines how these themes and patterns help understand an issue or phenomenon in a deeper way for answering the research questions at hand. Qualitative analysis emphasises 'sense-making' or understanding a phenomenon rather than predicting or explaining. There are different types of QDA methods, but in this research, we only clarify the thematic analysis, which is widely used by qualitative researchers, who collect data by interviews or focus group discussions or other processes. 
Themes are constructed patterns (or meanings) derived from different data collection methods collected from a data set. Themes answer a research question as opposed to mere summaries or categorisations of codes. According to Braun and Clarke (2006), thematic analysis entails searching across a data set to recognise, analyse, and report repeated or frequent patterns. It not only describes data but also interprets the process of selecting codes or categories in developing themes.

It should be well understood when to use thematic analysis. Thematic analysis is suitable when a researcher seeks to understand a set of thoughts, experiences, or behaviours across a data set (Braun \& Clarke, 2012). This analysis is applicable to search for a shared or common meaning in the data set. Therefore, this method is not suitable for examining unique meanings or experiences from a single person or data set (Kiger \& Varpio, 2020).

Now, we will be discussing how to develop themes for the analysis. However, forming codes or categories represents the heart of QDA. The two terms, i.e. codes and categories, are used interchangeably (Creswell \& Poth, 2018). However, in developing themes, we would like to show you the model of thematic analysis proposed by Braun and Clarke $(2006,2012)$ as mentioned in the book by Dawson (2019).

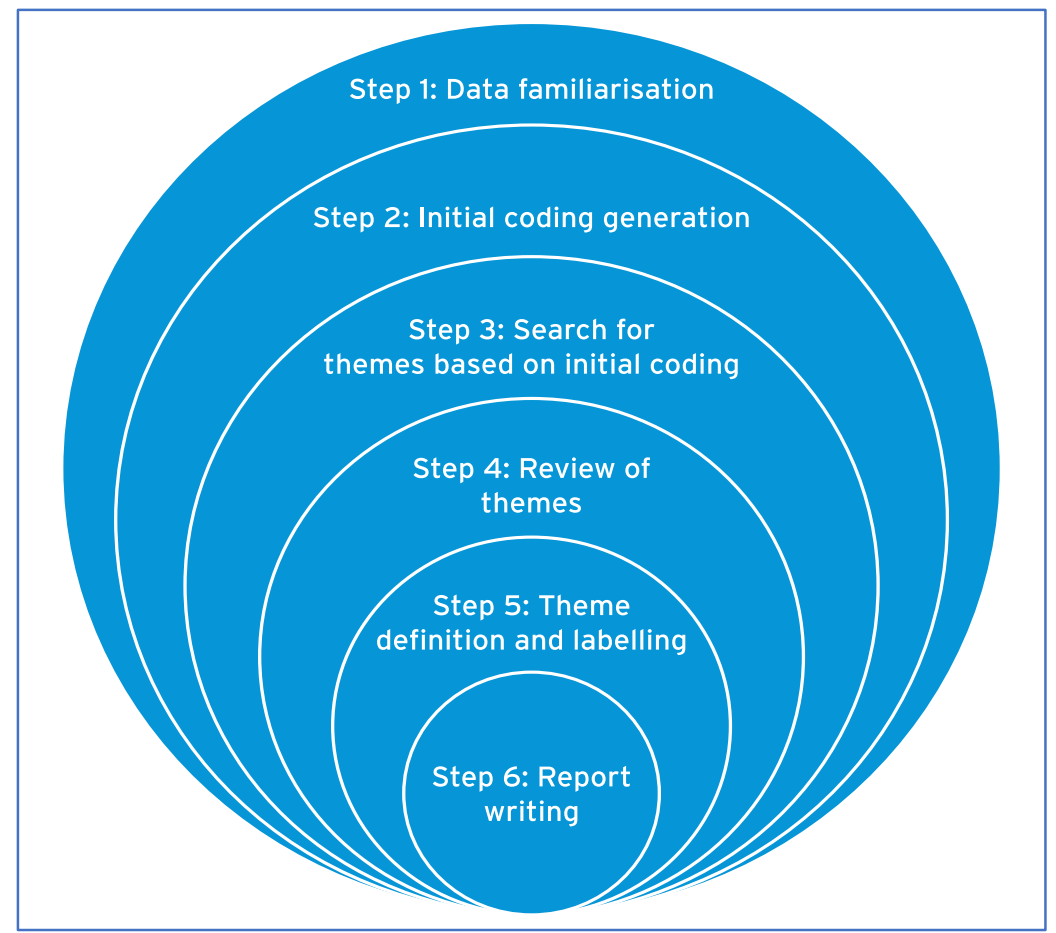

Figure 3: Braun and Clarke's (2006, 2012) Model of Thematic Analysis Source: As mentioned in the book of Dawson, (2019)

According to the model illustrated in Figure 3, in stage 1, you should be familiar with the close details of the transcript or whatever other texts are to be used. Read the same a few times and get acquainted with the text or transcript that will lead to the second step. In stage 2 , you will develop initial codes. Here, you should know that we understand that a higher analytic effort contributes to better research. In this regard, the initial and formal analysis step in the thematic analysis is the line-by-line coding of the data. The codes are not themes but help the researcher generate themes. The coding is little different than a label to explain the contents of one or two lines of the transcripts. In this stage, the researcher should look to capture a segment of the text's essence. This is called the initial stage, guided by the fuller 
picture and not simply one or two sentences in the texts. Furthermore, these codes are not something that can be generated when a researcher looks at their data. Therefore, a researcher has to create codes trying out ideas concerning their data.

Once the codes are ready, a researcher can start with stage 3, searching for themes based on initial coding. Here, the researcher should look for the patterns by asking 'what are the patterns among the codes?' For example, if codes were cat, orange, lion, potato, banana, tiger, and pumpkin, a researcher might suggest only two categories - vegetables and animals. However, another researcher may create another category (e.g. fruit) with additional code. These categories or groups are pretty much what a theme is. Therefore, it is crucial to ensure the categories are developed efficiently.

Stage 4 is called reviewing themes. In this stage, the researcher needs to organise the data around the themes to finalise the theme for the next step. The researcher would be able to amend the mistake in terms of themes. For example, a theme could be developed, but there is very little data to support the theme, which should be modified or abandoned in this stage.

Step 5 is called the theme definition and labelling. In this stage, the researcher has to bear in mind that the extent to which a particular theme is identified should be different conceptually from all of the other themes. It is possible when the researcher reviews the themes continuously according to the codes and categories. Thus, the researcher would be able to define and label every theme differently, thereby obtaining meaningful answers to the research questions.

Step 6, the last stage is report writing, which can be interpreted as the final stage of data analysis. It is the step wherein the researcher should polish and modify the analysis according to the problems. However, all the steps mentioned in Figure 3 relate back to each other. Therefore, the strength of the research question initiated in the beginning of a study defines the success. However, every researcher should know that a research question can be reformulated several times during the analysis process and even at the stage of documenting the final report (Dawson, 2019).

Finally, it is essential to note that researchers build detailed descriptions, apply codes, develop themes or dimensions, and provide an interpretation in light of their views or perspectives on the literature (Creswell \& Poth, 2018). Detailed description means that authors describe what they see (as mentioned in Creswell \& Poth, 2018). Thus, what a novice researcher finds or observes in the transcripts might be different from the observation of an expert. However, you will have to be an expert, and therefore, you have to continue the practice. In this regard, continuous development is possible once a researcher does hard work in a regular process. So far, we have known the QDA strategies, but what about the software to analyse? The following section gives ideas and justification regarding some software for QDA.

\section{What are the Suitable Software for Qualitative Data Analysis?}

Qualitative data can be analysed manually specially when the data are collected from a small sample. However, there are different software that can be used for QDA. In this regard, some standard software programs, such as QDA Miner, NVivo, ATLAS.ti, Quirkos, and Microsoft Word are widely used to analyse the rich qualitative data in qualitative research methods (Miles \& Huberman, 1984, Woods et al., 2016; Islam et al., 2020). It is vital to note that qualitative analysis is heavily dependent on analytic, integrative skills and personal knowledge of the researcher of the social context where the data is collected that may lead to personal bias. However, determination, honesty, and collection of data from various sources and ignoring researchers' existing knowledge regarding natural or new phenomenon 
being explored can effectively avoid biases in the data analysis process (Quilan, 2011; Islam, 2019). For example, the social context in Western societies and South Asian Societies or Middle East Societies are not the same. Therefore, the researcher should have enough awareness regarding social contexts to avoid bias in the data analysis process. However, in preventing biases in qualitative research, the telephone method has been supported in contemporary studies i.e. Vogl (2013) and Farooq and de Villiers (2017). Therefore, the researchers are also advised to use a telephone for interviewing the participant that would allow them to avoid bias in qualitative data collection (Farooq \& de Villiers, 2017).

However, above-mentioned software i.e. QDA Miner, NVivo, ATLAS.ti, Quirkos, and Microsoft Word are being used widely in the QDA process because of their distinctive advantages. Therefore, researchers are advised to read the features, advantages, and disadvantages of those software before using one in their research. In this regard, Lewis (2004) argued that NVivo 2.0 and ATLAS.ti 5.0 are among the best available and possibly most practical QDA tools (Islam, 2019; Dalkin et al., 2021). Both of these tools enable a researcher to associate labels or codes with amounts of text, pictures, videos, or sounds to search or explore these codes for patterns (Lewis, 2004).

Furthermore, both of these tools help build classifications of codes that mirror testable models of the conceptual structure of the fundamental raw data (Lewis, 2004). Moreover, both of these tools are remarkably flexible programs that can be applied in different applications. Furthermore, both also are Microsoft Windows-based products, which could be challenging to use in MacOS; therefore, these are the most popular QDA software among researchers worldwide.

\section{When and How to Reach the Saturation Point in Qualitative Research}

According to Saunders et al. (2018), saturation has attained widespread recognition and acceptance as a methodological standard or principle in qualitative research. It is used to determine when there is adequate data from a study to develop a robust and valid understanding of the study phenomenon (Hennink \& Kaiser, 2019).

However, it is important to share that prior to 2006, no researcher argued on the saturation in qualitative research. However, Guest et al. (2006) opened the Pandora's box and geared up a subject previously treated superficially. According to Guest et al. (2006), data saturation refers to a point in the research process when no new information is discovered from data and redundancy of the same findings begins and continues in the data analysis process. This redundancy signals to researchers that data collection may be ceased (as cited in Faulkner \& Trotter, 2017). Thus, when you do not find anything new from the data, you have reached the 'saturation point' in the qualitative research (Otmar et al., 2011; Jassim \& Whitford, 2014; Kazley et al., 2015).

Our suggestion to every researcher is that you should transcribe the interview or focus group discussion or any event you observe for your research immediately such that the raw data can be coded or categorised to develop themes. If you do the same one after one, you will identify the same codes, categories, or themes emerging from the last transcripts. When you find that the last transcripts of a series of interviews show similar findings, you can stop data collection and argue that you have reached the saturation point. Some scholars may ask how many interviews are required to reach the saturation point in qualitative research. In this regard, we should honestly endeavour to show that it is reached when the $(\mathrm{n}+1)$ th interview does not bring anything new than the $(\mathrm{n})$ th interview. Thus, we can argue that saturation point is found in qualitative research using interviews as data collection methods. It is because data collection using interviews is influenced by a number of factors, 
which include age, gender, geography, culture, and confidentiality. These factors differently impact the interviewer and interviewee; therefore, if we give a magic number for saturation point, it would ignore the influence of those factors.

\section{Concluding Remarks and Future Directions}

Overall, this study includes only a discussion regarding qualitative research method, research approaches, sampling strategy, sample size, interview method, saturation, and data analysis. The researchers have mentioned all the justifications and analysis in a succinct manner, which is one of the key limitations of the study. Furthermore, only few key methods under the qualitative research domain have been discussed in this study. Therefore, the readers should use the insights from this study cautiously. However, future studies can elaborate the discussions of the study and some methodological areas such as research philosophy, other data collection and analysis methods, and so on.

\section{References}

Anderson, C. (2010). Presenting and evaluating qualitative research. American Journal of Pharmaceutical Education, 74(8).

Braun, V., \& Clarke, V. (2006). Using thematic analysis in psychology. Qualitative Research in Psychology, 3, 77-101.

Braun, V., \& Clarke, V. (2012). Thematic analysis. In H. Cooper, P. M. Camic, D. L. Long, A. T. Panter, D. Rindskopf, \& K. J. Sher (Eds.), APA handbook of research methods in psychology, Vol. 2. Research designs: Quantitative, qualitative, neuropsychological, and biological (pp. 57-71). American Psychological Association.

Cleland, J. A. (2017). The qualitative orientation in medical education research. Korean Journal of Medical Education, 29(2), 61.

Dalkin, S., Forster, N., Hodgson, P., Lhussier, M., \& Carr, S. M. (2021). Using computer assisted qualitative data analysis software (CAQDAS; NVivo) to assist in the complex process of realist theory generation, refinement and testing. International Journal of Social Research Methodology, 24(1), 123-134.

Dawson, C. (2019). Introduction to Research Methods: A Practical Guide for Anyone Undertaking a Research Project, $5^{\text {th }}$ ed, Robinson.

Evans, C., \& Lewis, J. (2018). Analysing semi-structured interviews using thematic analysis: Exploring voluntary civic participation among adults (pp. 1-6). SAGE Publications Limited.

Faulkner, S. L., \& Trotter, S. P. (2017). Theoretical saturation. The International Encyclopedia of Communication Research Methods, pp. 1-2.

Farooq, M. B. and de Villiers, C. (2017), Telephonic qualitative research interviews: when to consider them and how to do them, Meditari Accountancy Research, 25(2).

Fraenkel, J. R., Wallen, N. E., \& Hyun, H. H. (2015). How to design and evaluate research in education. 9" ed, Singapore, Mc Graw Hill

Gill, P., Stewart, K., Treasure, E., \& Chadwick, B. (2008). Methods of data collection in qualitative research: interviews and focus groups. British Dental Journal, 204(6), 291-295.

Guest, G., Bunce A., \& Johnson, L. (2006). How many interviews are enough? An experiment with data saturation and variability. Field Methods, 18(1), 59-82.

Hennink, M. M., \& Kaiser, B. N. (2019). Saturation in Qualitative Research. SAGE Publications Limited. 
Islam, M. A., Hunt, A., Jantan, A. H., Hashim, H., \& Chong, C. W. (2020). Exploring challenges and solutions in applying green human resource management practices for the sustainable workplace in the ready-made garment industry in Bangladesh. Business Strategy \& Development, 3(3), 332-343.

Islam, M. A. (2019). Challenges, Barriers and Coping Strategies Experienced by Women Leaders in Public Universities of Malaysia. [Doctoral Thesis, Universiti Putra Malaysia (UPM)] UPM Repository. http://psasir.upm.edu.my/

Jassim, G. A., \& Whitford, D. L. (2014). Understanding the experiences and quality of life issues of Bahraini women with breast cancer. Social Science \& Medicine, 107, 189195.

Kiger, M. E., \& Varpio, L. (2020). Thematic analysis of qualitative data: AMEE Guide No. 131. Medical Teacher, 42(8), 846-854.

Lewis, R. B. (2004). NVivo 2.0 and ATLAS. ti 5.0: A comparative review of two popular qualitative data-analysis programs. Field Methods, 16(4), 439-464.

May, K. A. (1991). Interview techniques in qualitative research: Concerns and challenges. Qualitative Nursing Research: A Contemporary Dialogue, 188-201.

Merriam, S. B., \& Tisdell, E. J. (2015). Qualitative research: A guide to design and implementation. John Wiley \& Sons.

Miles M. B., Huberman A. M. (1984). Qualitative Data Analysis: A Sourcebook of New Methods. Newbury Park, CA: Sage Publications.

Otmar, R., Kotowicz, M. A., Nicholson, G. C., \& Pasco, J. A. (2011). Methodological reflections on using pilot data from fracture patients to develop a qualitative study. BMC Research Notes, 4(1), 1-7.

Patton, M. Q. (2015). Qualitative research \& evaluation methods: Integrating theory and practice. $4^{\text {th }}$ ed. Thousand Oaks, CA: SAGE.

Quilan, C., (2011) Business Research Methods. 1" ed, Hampshire, Cengage Learning.

Saunders, M., Lewis, P., \& Thornhill, A. (2009). Research methods for business students. Pearson education.

Schmidt, C. (2004). The analysis of semi-structured interviews. A companion to qualitative research, 253, 258.

Saunders, B., Sim, J., Kingstone, T., Baker, S., Waterfield, J., Bartlam, B., Burroughs, H. \& Jinks, C. (2018). Saturation in qualitative research: exploring its conceptualization and operationalization. Quality \& Quantity, 52(4), 1893-1907.

Silverman, D. (2000). Doing Qualitative Research, Sage Publications.

Stake, R. E. (2010). The art of case study research. Thousand Oaks, CA: Sage.

Vogl, S. (2013), "Telephone versus face-to-face interviews: mode effect on semi-structured interviews with children", Sociological Methodology, 43(1), pp. 133-177.

Wiley \& Son. Tracy, S. J. (2013). Qualitative research methods: Collecting evidence, crafting analysis, communicating impact. Hoboken, NJ: Wiley-Blackwell.

Woods, M., Paulus, T., Atkins, D. P., \& Macklin, R. (2016). Advancing qualitative research using qualitative data analysis software (QDAS)? Reviewing potential versus practice in published studies using ATLAS. ti and NVivo, 1994-2013. Social Science Computer Review, 34(5), 597-617.

Yin, R. K. (2014). Case study research: Design and methods (5 ${ }^{\text {th }}$ ed.). Thousand Oaks, CA: Sage.

This work is licensed under a Creative Commons I Attribution-NonCommercial 3.0 Unported License. 